\title{
Correction to: Gestational hypertensive disorders and retinal microvasculature: the Generation R Study
}

Laura Benschop ${ }^{1 *}$, Sarah Schalekamp-Timmermans ${ }^{1}$, Jeanine E. Roeters van Lennep ${ }^{2}$, Vincent W. V. Jaddoe ${ }^{3,4}$, Tien Yin Wong ${ }^{6}$, Carol Y. Cheung ${ }^{6,7}$, Eric A. P. Steegers ${ }^{1}$ and M. Kamran Ikram³

\section{Erratum}

The original article [1] contained an error whereby the senior author, M. Kamran Ikram's name was mistakenly interchanged.

This error has now been corrected.

\begin{abstract}
Author details
'Department of Obstetrics and Gynecology, Erasmus Medical Center, Wytemaweg 80, PO Box 2040, 3000, CA, Rotterdam, The Netherlands. 2Department of General Medicine, Erasmus Medical Center, Rotterdam, The Netherlands. ${ }^{3}$ Department of Epidemiology, Erasmus Medical Center, Rotterdam, The Netherlands. ${ }^{4}$ Department of Pediatrics, Erasmus Medical Center, Rotterdam, The Netherlands. ${ }^{5}$ Department of Neurology, Erasmus Medical Center, Rotterdam, The Netherlands. ${ }^{6}$ Singapore Eye Research Institute, Singapore National Eye Centre, Singapore, Republic of Singapore. ${ }^{7}$ Department of Ophthalmology and Visual Sciences, The Chinese University of Hong Kong, Shatin, Hong Kong.
\end{abstract}

Received: 20 September 2017 Accepted: 20 September 2017 Published online: 06 October 2017

\section{References}

1. Benschop L, et al. Gestational hypertensive disorders and retinal microvasculature: the generation R study. BMC Med. 2017;15:153.

\footnotetext{
*Correspondence: h.benschop@erasmusmc.nl

'Department of Obstetrics and Gynecology, Erasmus Medical Center, Wytemaweg 80, PO Box 2040, 3000, CA, Rotterdam, The Netherlands Full list of author information is available at the end of the article
} 